\title{
Full Phase Diagram of Active Brownian Disks: From Melting to Motility-Induced Phase Separation
}

\author{
Pasquale Digregorio, ${ }^{1}$ Demian Levis,,${ }^{2,3}$. Antonio Suma, ${ }^{4,5}$ Leticia F. Cugliandolo, ${ }^{6}$ \\ Giuseppe Gonnella, ${ }^{1}$ and Ignacio Pagonabarraga ${ }^{2,3}$ \\ ${ }^{1}$ Dipartimento di Fisica, Università degli Studi di Bari and INFN, Sezione di Bari, via Amendola 173, Bari, I-70126, Italy \\ ${ }^{2}$ CECAM Centre Européeen de Calcul Atomique et Moléculaire, Ecole Polytechnique Fédérale de Lausanne, Batochimie, \\ Avenue Forel 2, 1015 Lausanne, Switzerland \\ ${ }^{3}$ UBICS University of Barcelona Institute of Complex Systems, Martí i Franquès 1, E08028 Barcelona, Spain \\ ${ }^{4}$ SISSA-Scuola Internazionale Superiore di Studi Avanzati, Via Bonomea 265, 34136 Trieste, Italy \\ ${ }^{5}$ Institute for Computational Molecular Science, Temple University, Philadelphia, Pennsylvania 19122, USA \\ ${ }^{6}$ Sorbonne Université, Laboratoire de Physique Théorique et Hautes Energies, CNRS UMR 7589, 4 Place Jussieu, \\ 75252 Paris Cedex 05, France
}

(Received 25 May 2018; published 31 August 2018)

\begin{abstract}
We establish the complete phase diagram of self-propelled hard disks in two spatial dimensions from the analysis of the equation of state and the statistics of local order parameters. The equilibrium melting scenario is maintained at small activities, with coexistence between active liquid and hexatic order, followed by a proper hexatic phase, and a further transition to an active solid. As activity increases, the emergence of hexatic and solid order is shifted towards higher densities. Above a critical activity and for a certain range of packing fractions, the system undergoes motility-induced phase separation and demixes into low and high density phases; the latter can be either disordered (liquid) or ordered (hexatic or solid) depending on the activity.
\end{abstract}

DOI: 10.1103/PhysRevLett.121.098003

Active materials are out-of-equilibrium systems in which the dynamics of their elements break detailed balance [1]. Examples can be found in living systems, e.g., the collective motion of large animal groups $[2,3]$, bacteria swarming [4], and the formation of traveling fronts of actin filaments [5], as well as in synthetic ones, like self-propelled grains [6] or self-catalytic colloidal suspensions [7]. Despite such diversity, the emergence of activity-induced collective behavior is captured by minimal models that yield accurate descriptions and shed light on their universal character. A key example is the active Brownian particles (ABP) model, which considers spherical self-propelled particles with only excluded volume interactions [8-13]. A hallmark of active particle systems is that at high enough density and activity, self-propulsion triggers a motility-induced phase separation (MIPS) into a low-density gas in coexistence with a high-density drop [10-17], resembling the equilibrium liquid-gas transition but in the absence of cohesive forces and without a thermodynamic support $[18,19]$.

Although active particles can in principle move in three dimensions (3D), in most experimental set-ups they are confined to two dimensions (2D). Most studies of 2D ABP focused on MIPS, and they have therefore been largely restricted to intermediate densities [10-19]. In contrast, their solidification, or melting, has received little attention [20-22], and the connection between the high Pe behavior and the equilibrium physics as $\mathrm{Pe} \rightarrow 0$ has been, surprisingly, disregarded. In particular, the fate of 2D melting (with its intermediate hexatic phase) under active forces, has been investigated for dumbbell systems [23], where MIPS is continuously connected to the passive liquidhexatic coexistence. This result shed new light on the very nature of MIPS, and it showed the importance of exploring the full phase diagram at high densities. In this Letter, we address this issue in the paradigmatic ABP model.

Melting in 2D is a fundamental problem that has remained elusive despite decades of intensive research [24,25]. The transition was initially claimed to be first order [26] and later argued to follow a different scenario, with an intermediate hexatic phase, separated by continuous transitions mediated by the unbinding of defects [27-29]. More recently, numerical simulations [30-32], followed by experiments on colloidal monolayers [33], clarified the picture. They indicate that melting of passive hard disks takes place in two steps: as the packing fraction is increased, a first-order transition between the liquid and hexatic phases occurs, followed by a continuous Berezinskii-Kosterlitz-Thouless (BKT) transition between the hexatic and the solid. The hexatic phase exhibits quasi-long-range orientational order and short-range positional one, while the solid phase has quasi-long-range positional and long-range orientational order. Liquid and hexatic phases coexist close to the liquid phase, within a narrow interval of packing fractions. 


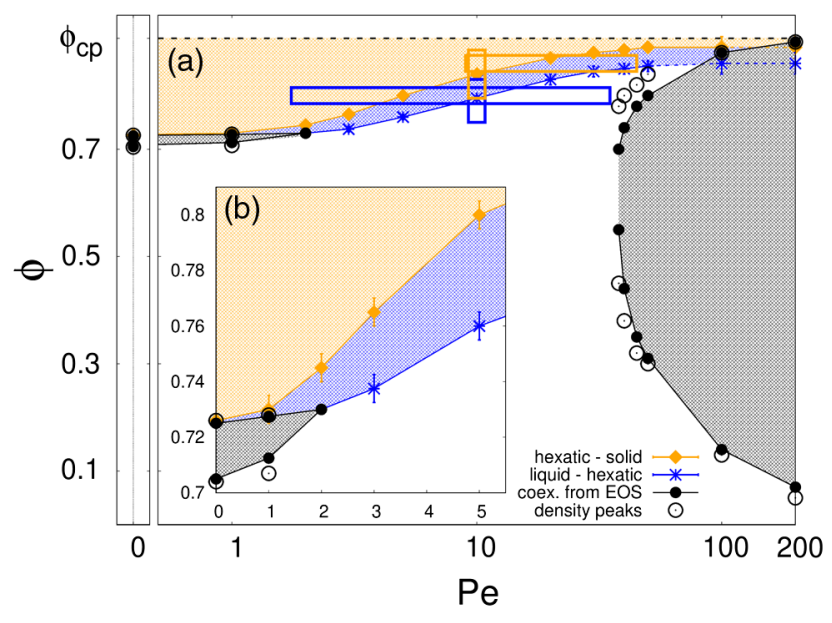

FIG. 1. Pe- $\phi$ phase diagram of ABP. In the inset, an enlargement over the low Pe-high $\phi$ regime highlights the connection with $2 \mathrm{D}$ melting. In the black area there is coexistence, in the blue hexatic order and the orange one is an active solid phase. The black filled (open) points were obtained from pressure (density distribution) measurements; the blue stars from the orientational correlations and the orange symbols from the spatial correlations decay. Although narrow, the blue area persists for $\mathrm{Pe} \rightarrow 0$ and broadens as Pe increases. The solid boxes highlight the parameters used to measure the correlations in Figs. 3 and 6.

Here, we examine how activity affects the phase behavior of 2D systems of isotropic particles (i.e. with no alignment interactions), from the dilute regime to close packing $\left(\phi_{\mathrm{cp}} \approx 0.91\right)$. We establish the complete phase diagram of 2D ABP spanning a broad range of activities, see Fig. 1. We show that the two-step melting scenario at $\mathrm{Pe}=0$ is maintained at finite but small activity, with a coexistence region between active liquid and hexatic phases (black area). Above, an active hexatic phase exists for all the explored activities (blue sector). Strikingly, active disks arrange in a hexatic phase in a larger density range than passive ones. At higher densities, orientational longrange and positional quasi-long-range order emerge for any activity, signaling the presence of an active solid phase (orange region). The liquid-hexatic and hexatic-solid transitions shift towards higher densities with Pe, meaning that activity destabilizes the ordered phases. At high enough activity (Pe $\gtrsim 35$ ), we identify the boundaries of MIPS using both pressure measurements and density distributions (black and white symbols). The MIPS region broadens as activity increases, and it eventually crosses the hexatic and solid transition lines. Such results show that (i) MIPS prevails over the hexatic and solid phases, and (ii) MIPS generates a phase separation between a dilute and a highdensity phase, which can either be liquid, hexatic, or solid, as activity is increased.

We consider $N$ overdamped ABP, in a square box with volume $V=L^{2}$ and periodic boundary conditions. They self-propel under a constant modulus force $F_{\text {act }}$ along $\mathbf{n}_{i}=\left(\cos \theta_{i}(t), \sin \theta_{i}(t)\right)$ and obey

$$
\gamma \dot{\mathbf{r}}_{i}=F_{\mathrm{act}} \mathbf{n}_{i}-\nabla_{i} \sum_{j(\neq i)} U\left(r_{i j}\right)+\boldsymbol{\xi}_{i}, \quad \dot{\theta}_{i}=\eta_{i},
$$

with $\mathbf{r}_{i}$ the position of the center of the $i$ th particle, $r_{i j}=$ $\left|\mathbf{r}_{i}-\mathbf{r}_{j}\right|$ the interparticle distance, and a short-ranged repulsive potential, $U(r)=4 \varepsilon\left[(\sigma / r)^{64}-(\sigma / r)^{32}\right]+\varepsilon$ if $r<\sigma_{d}=2^{1 / 32} \sigma$ and 0 otherwise. The terms $\xi$ and $\eta$ are zero-mean Gaussian noises that verify $\left\langle\boldsymbol{\xi}_{i}(t) \boldsymbol{\xi}_{j}\left(t^{\prime}\right)\right\rangle=$ $2 \gamma k_{B} T \delta_{i j} \delta\left(t-t^{\prime}\right) \mathbf{1}$ and $\left\langle\eta_{i}(t) \eta_{j}\left(t^{\prime}\right)\right\rangle=2 D_{\theta} \delta_{i j} \delta\left(t-t^{\prime}\right)$. The units of length, time, and energy are given by $\sigma_{d}, \tau=D_{\theta}^{-1}$ and $\varepsilon$, respectively. We fix $D_{\theta}=3 \gamma k_{B} T / \sigma_{d}^{2}$ and vary the packing fraction $\phi=\pi \sigma_{d}^{2} N /(4 V)$ and Péclet number $\mathrm{Pe}=F_{\text {act }} \sigma_{d} /\left(k_{B} T\right)$ by tuning $L$ and $F_{\text {act }}$ at fixed $\gamma=10$ and $k_{B} T=0.05$. The integration of Eqs. (1) used the velocity Verlet algorithm implemented in LAMMPS [34,35]. Simulations ran with $N=256^{2}$ particles, scanning the parameter space $\phi \in[0: 0.9]$ and $P e \in[0: 200]$. With less $\left(N=128^{2}\right)$ and more $\left(N=512^{2}\right)$ particles we explored finite size effects.

The equation of state.-Our first estimate of the phase boundaries is given by the $\phi$ dependence of the mechanical pressure $[18,36]$

$\Delta P=\frac{F_{\mathrm{act}}}{2 V} \sum_{i}\left\langle\mathbf{n}_{i} \cdot \mathbf{r}_{i}\right\rangle-\frac{1}{4 V} \sum_{i, j}\left\langle\nabla_{i} U\left(r_{i j}\right) \cdot\left(\mathbf{r}_{i}-\mathbf{r}_{j}\right)\right\rangle$

with $\Delta P=P-P_{G}$ and $P_{G}=N k_{B} T / V$ the ideal gas pressure. The first term, $P_{\text {act }}$, quantifies the effect of $F_{\text {act }}$, the so-called active or swim pressure [37,38]. The second one, $P_{\text {int }}$, is the standard virial term due to particle interactions. The definition in Eq. (2) is a state function for isotropic ABP such that $P(\phi)$ defines an equation of state [38]. (This does not hold generically in active systems for which the pressure can, for instance, depend on the details of the interaction between the particles and the confining walls [39].) In the dilute limit, we recover the ideal gas law $P V=N k_{B} T_{\text {eff }}=N k_{B} T\left(1+\mathrm{Pe}^{2} / 6\right)$, at an effective temperature that is compatible with the one that stems from the fluctuation-dissipation relation in the late diffusive regime [40-42].

The equation of state for zero and weak Pe is shown in Fig. 2(a). $P(\phi)$ is roughly flat in a narrow $\phi$ interval for $\mathrm{Pe} \lesssim 3$. A zoom over this area in the $\mathrm{Pe}=1$ case evidences a double loop structure characteristic of phase coexistence, see Fig. 2(b). Although the equal-area Maxwell construction that allows us to directly extract the binodals cannot be readily applied for $\mathrm{Pe}>0[19,38]$, we use it by extension of the passive disks analysis [31], as a first identification of the coexistence region (black dots in Fig. 1). Beyond $\mathrm{Pe}=3$, we do not find evidence for coexistence until the high-Pe regime where MIPS is attained. For Pe $\gtrsim 35$, the $P(\phi)$ curves become flat in between two densities. Representative curves at $10 \leq \mathrm{Pe} \leq 50$ are displayed in Fig. 2(c). As it has been recently reported [18,36], at very 

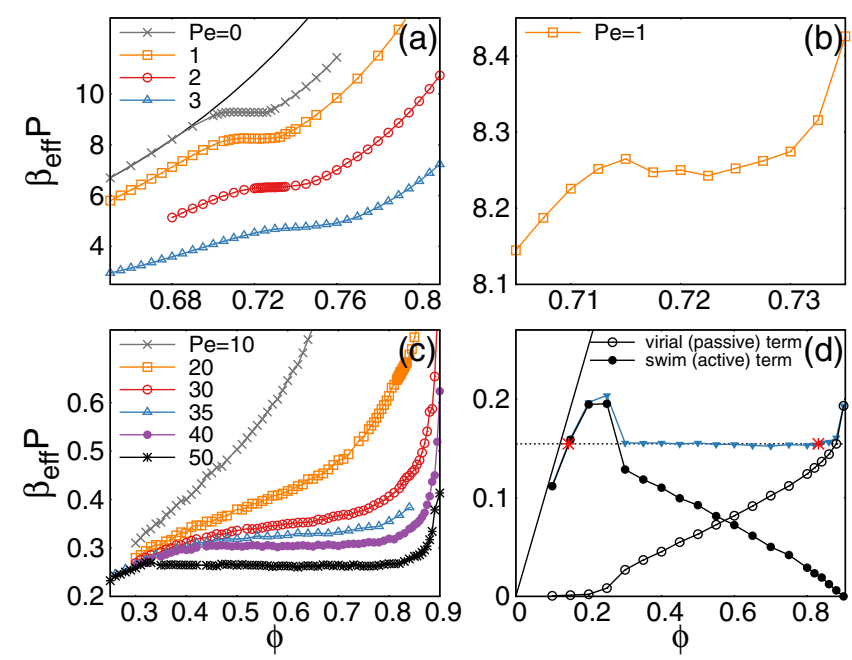

FIG. 2. Equation of state. (a) Numerical data for low Pe and the analytical form for passive hard disks (continuous line) [45]. (b) Details around the liquid-hexatic coexistence region for $\mathrm{Pe}=1$. (c) Data for intermediate Pe. (d) Swim $P_{\text {act }}$ and interaction $P_{\text {int }}$ contributions to $P$ in Eq. (2) from the gas to the solid at $\mathrm{Pe}=100$. Triangular symbols correspond to the total pressure. The ideal gas law is shown with a continuous line. The red symbols indicate the coexistence densities reported in Fig. 1.

high Pe, the pressure drops abruptly at the vicinity of MIPS, see Fig. 2(d), as a consequence of the existence of a metastability region with a very large nucleation barrier [18]. We obtain the limits of MIPS with an extrapolation of the flat part of $P(\phi)$ across the pressure jump (or spinodal), as illustrated in Fig. 2(d) for $\mathrm{Pe}=100$. Previous numerical studies used the local density probability distribution functions (PDF) to locate the MIPS region, see e.g. $[11,23]$. For the sake of completeness, we searched for the limits of a double peak structure of these PDFs, finding the open symbols in Fig. 1, in very good agreement with the pressure measurements (see Figs. S1, S2, and S3 in the Supplemental Material (SM) [43] for further details).

Orientational order and the hexatic phase.-We put the orientational order to the test using the hexatic order parameter $\psi_{6}\left(\mathbf{r}_{j}\right)=N_{j}^{-1} \sum_{k=1}^{N_{j}} e^{\mathrm{i} 6 \theta_{j k}}$, where $\theta_{j k}$ is the angle formed by the segment that connects the center of the $j$ th disk and the one of its $k$ th (out of $N_{j}$ ) nearest neighbor (found with a Voronoi tessellation algorithm) and the $x$ axis. We studied its correlation function $g_{6}\left(r=\left|\mathbf{r}_{j}-\mathbf{r}_{k}\right|\right)=$ $\left\langle\psi_{6}\left(\mathbf{r}_{j}\right) \psi_{6}\left(\mathbf{r}_{k}\right)\right\rangle /\left\langle\psi_{6}^{2}\left(\mathbf{r}_{j}\right)\right\rangle$ and kurtosis or Binder parameter $U_{4}=1-\left\langle\psi_{6}^{4}\left(\mathbf{r}_{j}\right)\right\rangle /\left(3\left\langle\psi_{6}^{2}\left(\mathbf{r}_{j}\right)\right\rangle^{2}\right)$, see Figs. 3 and 4, respectively. We use the change of behavior of $g_{6}(r)$, from exponential (active liquid, in black) to algebraic $r^{-\eta}$ (active hexatic, in blue), as a criterion to locate the hexatic transition (blue symbols in Fig. 1). In the hexatic (blue) region, the power law decay is maintained, with exponent $\eta$ taking a value close to the BKT $\eta=1 / 4$ at the transition but varying with $\phi$ and Pe. These data are compatible with the behavior of the Binder cumulant, $U_{4}$, that in the scale of the main

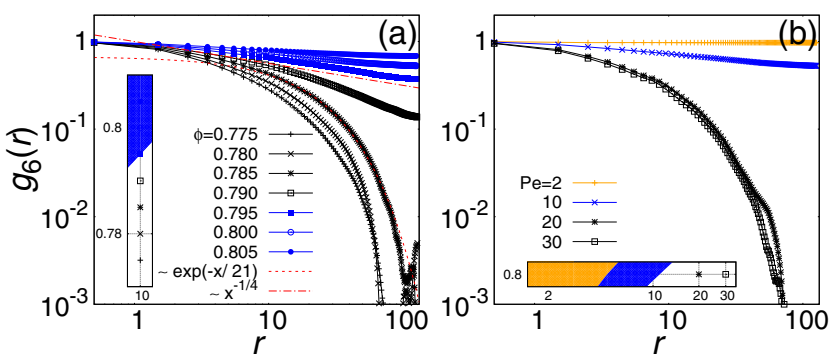

FIG. 3. Orientational correlations close to the active liquidhexatic transition for varying densities at $\mathrm{Pe}=10$ (a) and different $\mathrm{Pe}$ at $\phi=0.80$ (b). The vertical and horizontal boxes represent the location of the parameters in the phase diagram (see Fig. 1). An exponential fit to the liquid data and an algebraic decay with power $\eta=1 / 4$ are shown in (a).

panel in Fig. 4 has a common intersection point, proving the transition. The inset enlargement shows a weak remanent $N$ dependence that would be compatible with a first order phase transition [46,47]; however, the accuracy of our data is not enough to draw such a conclusion and, moreover, a second order transition is consistent with the absence of phase coexistence found above $\mathrm{Pe} \approx 3$. As illustrated in Fig. 3(b), activity shifts the emergence of orientational quasi-longrange order to higher densities.

Orientational order and coexistence.-The maps of the local hexatic order parameter and the PDFs of its modulus, shown in Fig. 5, provide clues to understand the difference between the two sectors with phase separation at low and high Pe. Close to Pe $=0$ the PDF is bimodal, see Fig. 5(a), with two peaks of roughly the same height for this choice of parameters. The map in Fig. 5(b) proves the existence of a ramified but large (of the order of the system size) region with the same local hexatic order. During the dynamics, this region changes form, but the portion of the surface that it occupies remains stable. These results are in perfect correspondence with the data for the local densities (see the SM in [43]). In the MIPS region, instead, the map shows many different colors, associated with diverse local orientational ordering, that do not extend over a long distance, even at long times, see Fig. 5(c). During the time evolution, the color pattern changes considerably, with the breaking and recombination of blocks. Differences in

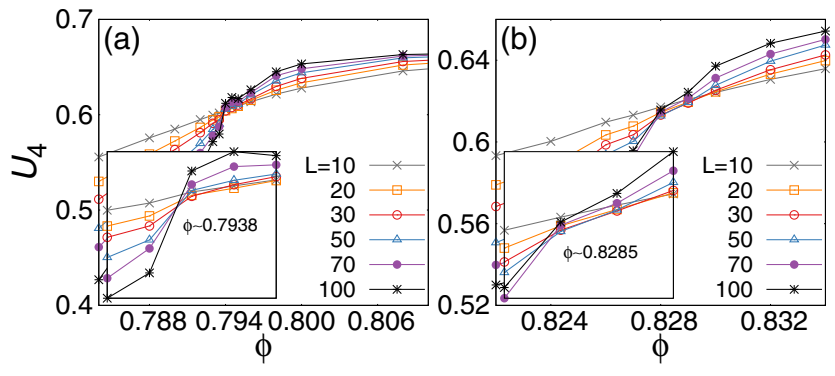

FIG. 4. The system size dependence of the Binder cumulant for $\mathrm{Pe}=10$ (a) and $\mathrm{Pe}=20$ (b). 

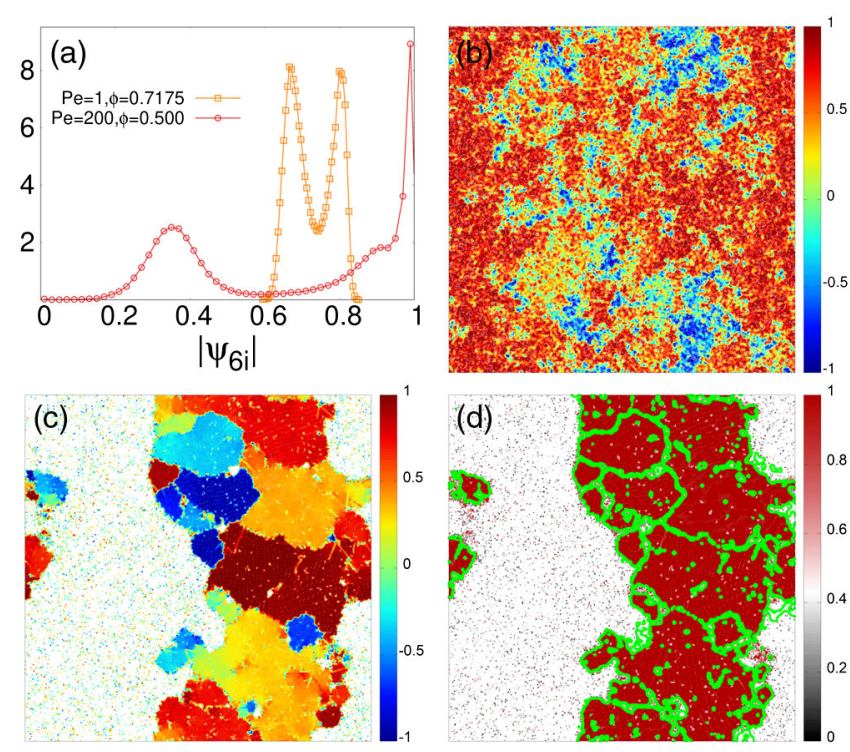

FIG. 5. (a) PDF of $\left|\psi_{6 i}\right|$ in the low Pe and MIPS coexistence region. (b), (c) Maps of $\psi_{6 i}$, projected into the mean orientation of the system [43], for the two cases considered in (a). (d) Snapshot showing $\left|\psi_{6 i}\right|$ and the interfaces between domains with different orientational order (in green).

the maps are translated into differences in the PDFs. The secondary peak, close to $\left|\psi_{6, j}\right|=0.9$ in Fig. 5(a), is due to the interfaces between areas with almost perfect orientational order. Additional maps in other sectors of the phase diagram, PDFs of $\left|\psi_{6, j}\right|$, correlation functions and global hexatic order parameter $\Psi=N^{-1}\left|\sum_{j} \psi_{6, j}\right|$ measurements are given in the SM.

Positional order and the solid phase.- Since it is hard to assert whether $g_{6}$ acquires long-range order or does not decay at the length scales of our finite-size box, we looked for solid quasi-long range positional order, that should be evidenced by an algebraic decay of

$$
C_{\mathbf{q}_{0}}(r)=\left\langle e^{\mathrm{i} \mathbf{q}_{0} \cdot\left(\mathbf{r}_{i}-\mathbf{r}_{j}\right)}\right\rangle,
$$

at the wave vector $\mathbf{q}_{0}$ at the maximum of the first diffraction peak of the structure factor $S(q)=N^{-1} \sum_{i, j} e^{\mathrm{iq} \cdot\left(\mathbf{r}_{i}-\mathbf{r}_{j}\right)}$. The change in the $C_{\mathbf{q}_{0}}$ decay, from exponential (hexatic) to algebraic (solid) for several Pe and $\phi$, see Fig. 6, yields the orange points in the phase diagram above which lies the solid. Activity introduces non-equilibrium fluctuations that destabilize order and melt the solid.

Summarizing, we established the full phase diagram of active Brownian hard disks, with active liquid, hexatic, and solid phases, as well as coexistence and MIPS.

First, we proved that the overall scenario of the 2D melting of passive disks is maintained for small-enough Pe. Weak activity acts as a perturbation that destabilizes passive order, similarly to what was found in [22] for a system of softer disks (no coexistence in the passive limit) evolved with Monte Carlo dynamics. This is shown by the fact that

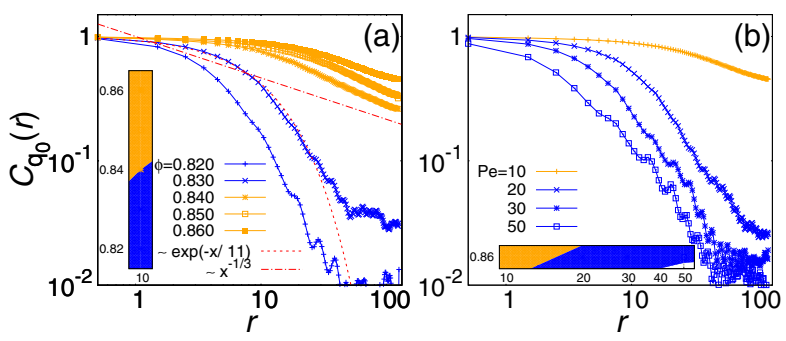

FIG. 6. Positional correlations $C_{\mathbf{q}_{0}}(r)$ close to the hexatic-solid transition for several values of $\phi$ at fixed $\mathrm{Pe}=10$ (a) and of Pe at fixed $\phi=0.86$ (b). In (a), we show an exponential and an algebraic decay with power $\eta=1 / 3$ (corresponding to the exponent predicted by the KTHNY theory [27-29]).

by increasing Pe, both the liquid-hexatic and hexatic-solid transitions shift to higher densities, and the liquid-hexatic coexistence region shrinks and eventually disappears. Such behavior can be due to the effective softness introduced by activity [quantified by the ratio between the active and potential forces $\left.\Gamma=\varepsilon /\left(\sigma_{d} F_{\text {act }}\right)\right]$, since, in equilibrium, particle softness reduces the liquid-hexatic coexistence region and eventually destroys it, rendering the hexaticliquid transition continuous [32].

At high Pe, the MIPS region opens up on top of the hexatic and solid transition lines (differently from what was shown in [22]) and the emergence of hexatic and solid order prevails. In most of the MIPS region, many finite-size patches with different hexatic order coexist at any moment, but the large activity makes them regularly rearrange via breaking and recombination. This phenomenon is very different from what happens at low Pe. Above the point at which the hexatic transition line crosses the MIPS binodal, activity triggers phase separation between a low-density gas and a high-density hexatic, or solid, at higher Pe.

The discontinuity between the coexistence regions for active Brownian disks is distinct from what was found for active dumbbells, for which the large Pe phase separation was continuously connected to the zero Pe one. This difference could be due to the fact that dumbbells have a nonconvex geometry that eases jamming and the formation of local orientational order. It would be interesting to study systems made of elements that interpolate between the disk and dumbbell geometries, and then see how the topology of the phase diagram transforms from the one in Fig. 1 to the one in [23].

To conclude, our results provide a firm basis to rationalize the phase behavior of dense active matter and understand how self-propulsion affects the liquid and solid phases of matter on general grounds. The scenario we established here could be experimentally tested in, for instance, monolayers of self-propelled Janus colloids.

This work was possible thanks to the access to the MareNostrum Supercomputer at the Barcelona Supercomputing Center (BSC), IBM Nextscale GALILEO at 
CINECA (Project No. INF16-fieldturb) under CINECAINFN agreement and Bari ReCaS e-Infrastructure funded by MIUR through PON Research and Competitiveness 2007-2013 Call 254 Action I. D. L. and I. P. acknowledge funding from the EU's Horizon 2020 programme under the Marie Sklodowska-Curie (IF) Grant Agreement No. 657517 and Ministerio de Economia y Competititividad (MINECO) and Departament d'Universitats, Recerca i Societat de la Informacio (DURSI) under Projects No. FIS2015-67837-P and No. 2017SGR-884, respectively. L. F. C. is a member of Institut Universitaire de France, thanks the Kavli Institute of Theoretical Physics (KITP) University of Santa Barbara for hospitality and L. Berthier, P. Choudhuri, C. Dasgupta, M. Dijkstra, and J. Klamser for useful discussions.

*demian.levis@epfl.ch

[1] M. C. Marchetti, J.-F. Joanny, S. Ramaswamy, T. B. Liverpool, J. Prost, M. Rao, and R. A. Simha, Rev. Mod. Phys. 85, 1143 (2013).

[2] I. D. Couzin and J. Krause, Adv. Study Behav. 32, 1 (2003).

[3] W. Bialek, A. Cavagna, I. Giardina, T. Mora, E. Silvestri, M. Viale, and A. M. Walczak, Proc. Natl. Acad. Sci. USA 109, 4786 (2012).

[4] H.-P. Zhang, A. Beer, E.-L. Florin, and H. L. Swinney, Proc. Natl. Acad. Sci. USA 107, 13626 (2010).

[5] V. Schaller, C. Weber, C. Semmrich, E. Frey, and A. R. Bausch, Nature (London) 467, 73 (2010).

[6] J. Deseigne, O. Dauchot, and H. Chaté, Phys. Rev. Lett. 105, 098001 (2010).

[7] C. Bechinger, R. Di Leonardo, H. Löwen, C. Reichhardt, and G. Volpe, Rev. Mod. Phys. 88, 045006 (2016).

[8] B. ten Hagen, S. van Teeffelen, and H. Löwen, J. Phys. Condens. Matter 23, 194119 (2011).

[9] P. Romanczuk, M. Bär, W. Ebeling, B. Lindner, and L. Schimansky-Geier, Eur. Phys. J. Spec. Top. 202, 1 (2012).

[10] Y. Fily and M. C. Marchetti, Phys. Rev. Lett. 108, 235702 (2012).

[11] G. S. Redner, M. F. Hagan, and A. Baskaran, Phys. Rev. Lett. 110, 055701 (2013).

[12] M. Cates and J. Tailleur, Europhys. Lett. 101, 20010 (2013).

[13] J. Bialké, H. Löwen, and T. Speck, Europhys. Lett. 103, 30008 (2013).

[14] J. Tailleur and M. E. Cates, Phys. Rev. Lett. 100, 218103 (2008).

[15] J. Stenhammar, D. Marenduzzo, R. J. Allen, and M. E. Cates, Soft Matter 10, 1489 (2014).

[16] J. Bialké, J. T. Siebert, H. Löwen, and T. Speck, Phys. Rev. Lett. 115, 098301 (2015).

[17] M. E. Cates and J. Tailleur, Annu. Rev. Condens. Matter Phys. 6, 219 (2015).

[18] D. Levis, J. Codina, and I. Pagonabarraga, Soft Matter 13, 8113 (2017).

[19] A. P. Solon, J. Stenhammar, M. E. Cates, Y. Kafri, and J. Tailleur, Phys. Rev. E 97, 020602 (2018).

[20] J. Bialké, T. Speck, and H. Löwen, Phys. Rev. Lett. 108, 168301 (2012).
[21] G. Briand and O. Dauchot, Phys. Rev. Lett. 117, 098004 (2016).

[22] J. U. Klamser, S.C. Kapfer, and W. Krauth, arXiv: 1802.10021.

[23] L. F. Cugliandolo, P. Digregorio, G. Gonnella, and A. Suma, Phys. Rev. Lett. 119, 268002 (2017).

[24] K. J. Strandburg, Rev. Mod. Phys. 60, 161 (1988).

[25] V. N. Ryzhov, E. E. Tareyev, Y. D. Fomin, and E. N. Tsiok, Phys. Usp. 60, 857 (2017).

[26] B. J. Alder and T.E. Wainwright, Phys. Rev. 127, 359 (1962).

[27] J. M. Kosterlitz and D. J. Thouless, J. Phys. C 6, 1181 (1973).

[28] B. I. Halperin and D. R. Nelson, Phys. Rev. Lett. 41, 121 (1978).

[29] A. Young, Phys. Rev. B 19, 1855 (1979).

[30] E. P. Bernard, W. Krauth, and D. B. Wilson, Phys. Rev. E 80, 056704 (2009).

[31] E. P. Bernard and W. Krauth, Phys. Rev. Lett. 107, 155704 (2011).

[32] S. C. Kapfer and W. Krauth, Phys. Rev. Lett. 114, 035702 (2015).

[33] A. L. Thorneywork, J. L. Abbott, D. G. A. L. Aarts, and R. P. A. Dullens, Phys. Rev. Lett. 118, 158001 (2017).

[34] S. Plimpton, J. Comput. Phys. 117, 1 (1995).

[35] The algorithm integrates the underdamped process corresponding to Eq. (1). We set the parameters to be in the overdamped limit (see [23] for details).

[36] R. G. Winkler, A. Wysocki, and G. Gompper, Soft Matter 11, 6680 (2015).

[37] S. C. Takatori, W. Yan, and J. F. Brady, Phys. Rev. Lett. 113, 028103 (2014).

[38] A. P. Solon, J. Stenhammar, R. Wittkowski, M. Kardar, Y. Kafri, M. E. Cates, and J. Tailleur, Phys. Rev. Lett. 114, 198301 (2015).

[39] A. P. Solon, Y. Fily, A. Baskaran, M. E. Cates, Y. Kafri, M. Kardar, and J. Tailleur, Nat. Phys. 11, 673 (2015).

[40] A. Suma, G. Gonnella, G. Laghezza, A. Lamura, A. Mossa, and L. F. Cugliandolo, Phys. Rev. E 90, 052130 (2014).

[41] D. Levis and L. Berthier, Europhys. Lett. 111, 60006 (2015).

[42] F. Ginot, I. Theurkauff, D. Levis, C. Ybert, L. Bocquet, L. Berthier, and C. Cottin-Bizonne, Phys. Rev. X 5, 011004 (2015).

[43] See Supplemental Material at http://link.aps.org/ supplemental/10.1103/PhysRevLett.121.098003, which includes Ref. [44], for further details on the phase diagram, including additional histograms of the local surface fraction and of $\left|\psi_{6 i}\right|$, several maps of $\psi_{6 i}$, additional hexatic correlation functions and the analysis of the global hexatic order parameter along with its finite-size behaviour.

[44] M. Engel, A. J. Anderson, S. C. Glotzer, M. Isobe, E. P. Bernard, and W. Krauth, Phys. Rev. E 87, 042134 (2013).

[45] J. Kolafa and M. Rottner, Mol. Phys. 104, 3435 (2006).

[46] K. Vollmayr, J. D. Reger, M. Scheucher, and K. Binder, Z. Phys. B 91, 113 (1993).

[47] H. Weber, D. Marx, and K. Binder, Phys. Rev. B 51, 14636 (1995). 